\title{
To fit Fermi's weak coupling constant with three gravitational constants
}

\author{
U. V. S. Seshavatharam ${ }^{1}$ and S. Lakshminarayana ${ }^{2}$ \\ ${ }^{1}$ Hon. Faculty, I-SERVE, S. No-42, Hitex Rd., Hitech city, Hyderabad-84, Telangana, India \\ ${ }^{2}$ Department of Nuclear Physics, Andhra University, Visakhapatnam-03, AP, India \\ Corresponding Emails: seshavatharam.uvs@gmail.com; vedakavi@serveveda.org; sln@auvsp.edu.in;
}

\begin{abstract}
By considering three virtual gravitational constants assumed to be associated with gravitational, electromagnetic and strong interactions, Fermi's weak coupling constant can be shown to be a natural manifestation of microscopic quantum gravity. As our approach is heuristic and completely different from the current methods of estimating the Newtonian gravitational constant, with reference to the call of 'Ideas lab 2016' organized by NSF, we appeal for inclusion of this theoretical work as a project under unification scheme.
\end{abstract}

Keywords: Final unification; Fermi’s weak coupling constant; Newtonian gravitational constant; Virtual electromagnetic gravitational constant; Virtual nuclear gravitational constant;

\section{Introduction}

The most desirable cases of any unified description are:

a) To implement gravity in microscopic physics and to estimate the magnitude of Newtonian gravitational constant.

b) To develop a model of microscopic quantum gravity.

c) To simplify the complicated issues of known physics.

d) To predict new effects, arising from a combination of the fields inherent in the unified description.

In this context, in our earlier publication [1] and references therein, we suggested the role of two new gravitational constants associated with strong and electromagnetic interactions. In this paper, we make a bold attempt to interrelate the Fermi's weak coupling constant [2,3] and Newtonian gravitational constant $[4,5,6]$ via the two proposed electromagnetic and nuclear gravitational constants. We would like to appeal that, with respect to String theory models, Quantum gravity models [7] and proposed assumptions, it is possible to show that, weak interaction is a natural manifestation of microscopic quantum gravity [3].

\section{Nomenclature and magnitudes}

1) $e \cong 1.602176565 \times 10^{-19} \mathrm{C}=$ Elementary charge

2) $m_{p} \cong 1.672621777 \times 10^{-27} \mathrm{~kg}=$ Rest mass of proton

3) $m_{e} \cong 9.10938291 \times 10^{-31} \mathrm{~kg}=$ Rest mass of electron

4) $\hbar \cong 1.054571726 \times 10^{-34} \mathrm{~J}$. sec. $=$ Reduced Planck's constant

5) $c \cong 2.99792458 \times 10^{8} \mathrm{~m} \cdot \mathrm{sec}^{-1}$.

6) $G_{s} \cong 3.32956081 \times 10^{28} \mathrm{~m}^{3} \mathrm{~kg}^{-1} \mathrm{sec}^{-2}=$ Estimated virtual gravitational constant associated with nuclear or strong interaction
7) $G_{e} \cong 2.374335472 \times 10^{37} \mathrm{~m}^{3} \mathrm{~kg}^{-1} \mathrm{sec}^{-2}=$ Estimated virtual gravitational constant associated with electromagnetic interaction

8) $G_{N} \cong(6.619386$ to 6.679856$) \times 10^{-11} \mathrm{~m}^{3} \mathrm{~kg}^{-1} \mathrm{sec}^{-2}=$ Estimated (virtual) gravitational constant associated with gravitational interaction

9) $G_{F} \cong 1.44021 \times 10^{-62} \mathrm{~J}^{3} \mathrm{~m}^{3}=$ Estimated Fermi's weak coupling constant

10) $R_{0} \cong\left(2 G_{s} m_{p} / c^{2}\right) \cong 1.239291 \times 10^{-15} \mathrm{~m}=$ Estimated nuclear charge radius

\section{Two basic assumptions of final unification}

In our earlier publication, we proposed the following two assumptions [1].

Assumption-1: Magnitude of the virtual gravitational constant associated with the electromagnetic interaction is, $G_{e} \cong 2.374335472 \times 10^{37} \mathrm{~m}^{3} \mathrm{~kg}^{-1} \mathrm{sec}^{-2}$ where,

$$
\left(\frac{m_{p}}{m_{e}}\right) \cong 2 \pi \sqrt{\frac{4 \pi \varepsilon_{0} G_{e} m_{e}^{2}}{e^{2}}}
$$

Assumption-2: Magnitude of the virtual gravitational constant associated with the strong interaction $[8,9]$ is, $G_{s} \cong 3.32956081 \times 10^{28} \mathrm{~m}^{3} \mathrm{~kg}^{-1} \mathrm{sec}^{-2}$ where,

$$
\hbar c \cong \sqrt{\left(G_{s} m_{p} m_{e}\right)\left(G_{e} m_{e}^{2}\right)}
$$




\section{Important and interesting relations}

\section{A) Ratio of rest mass of proton to electron:}

$$
\left(\frac{m_{p}}{m_{e}}\right) \cong\left(\frac{G_{s}}{G_{N}^{2 / 3} G_{e}^{1 / 3}}\right)^{\frac{1}{7}}
$$

B) Nuclear charge radius:

$$
R_{0} \cong \frac{2 G_{s} m_{p}}{c^{2}} \cong 1.239291 \times 10^{-15} \mathrm{~m}
$$

\section{C) Root mean square radius of proton:}

$$
R_{p} \cong \frac{\sqrt{2} G_{s} m_{p}}{c^{2}} \cong 0.8763111 \times 10^{-15} \mathrm{~m}
$$

This can be compared with the recommended value [2] of $(0.8751 \pm 0.0061) \times 10^{-15}$ 爉

D) Ground state potential energy of electron in Hydrogen atom:

$a_{0}$ being the Bohr radius, energy conservation point of view,

$$
\begin{gathered}
\frac{G_{e} m_{e}^{2}}{a_{0}} \cong\left(\frac{e^{2}}{4 \pi \varepsilon_{0}}\left(\frac{G_{s} m_{p}}{c^{2}}\right)^{-1}\right) \\
\rightarrow a_{0} \cong\left(\frac{4 \pi \varepsilon_{0} G_{e} m_{e}^{2}}{e^{2}}\right)\left(\frac{G_{s} m_{p}}{c^{2}}\right) \cong 5.3 \times 10^{-11} \mathrm{~m} \\
\left(E_{\text {pot }}\right)_{\text {ground }} \cong-\left(\frac{e^{2}}{4 \pi \varepsilon_{0} a_{0}}\right) \\
\cong-\left(\frac{e^{2}}{4 \pi \varepsilon_{0} G_{e} m_{e}^{2}}\right)\left(\frac{e^{2}}{4 \pi \varepsilon_{0}}\left(\frac{G_{s} m_{p}}{c^{2}}\right)^{-1}\right) \cong-27.2 \mathrm{eV}
\end{gathered}
$$

Note: Considering $\left(\frac{1}{2 n^{2}}\right)$ as a probability of finding electron in any orbit labeled with $n=1,2,3, .$. further research can be carried out.

\section{E) Characteristic atomic radius of Hydrogen atom:}

$$
R_{\text {hydrogen }} \cong \frac{2 \sqrt{\left(G_{s} G_{e}\right)} m_{\text {atom }}}{c^{2}} \cong 33 \text { picometers }
$$

where $m_{\text {atom }}$ is the unified atomic mass, $1.66054 \times 10^{-27} \mathrm{~kg}$. This can be compared with radius of hydrogen atom associated with covalent bond. (https://en.wikipedia.org/wiki/Covalent_radius)

\section{F) Neutron star mass and radius}

1) If $\left(M_{N S}, m_{n}\right)$ represent the masses of neutron star [10] and neutron, then,

$$
\frac{G_{N} M_{N S} m_{n}}{\hbar c} \approx \sqrt{\frac{G_{S}}{G_{N}}} \rightarrow M_{N S} \approx 3.175 M_{\odot}
$$

Note: By considering $\left(\frac{\hbar}{2}\right)$, mass of neutron star can be estimated to be $1.5875 M_{\odot}$. This is just greater than the famous Chandrasekhar mass limit of $1.4 M_{\odot}$.

2) If $R_{N S}$ represents the neutron star radius [11], then,

$$
\frac{R_{N S}}{\left(\sqrt{G_{s} \hbar / c^{3}}\right)} \approx \sqrt{\frac{G_{s}}{G_{N}}} \rightarrow R_{N S} \approx 8.06 \mathrm{~km}
$$

\section{Fitting Fermi's weak coupling constant and electron rest mass}

Fitting the gravitational constant with elementary physical constants is a very challenging issue. According to G. Rosi et al [3]: "There is no definitive relationship between $G_{N}$ and the other fundamental constants, and there is no theoretical prediction for its value, against which to test experimental results. Improving the precision with which we know $G_{N}$ has not only a pure metrological interest, but is also important because of the key role that $G_{N}$ has in theories of gravitation, cosmology, particle physics and astrophysics and in geophysical models".

In this context, we would like to stress that, by considering the Fermi's weak coupling constant, in a verifiable approach, it is certainly possible to explore the back ground physics of the role of the Newtonian gravitational constant in microscopic physics. It may be noted that, according to Roberto Onofrio [3],

1) Weak interactions are peculiar manifestations of quantum gravity at the Fermi scale.

2) Fermi's weak coupling constant is related with the Newtonian constant of gravitation.

3) At atto-meter scale, Newtonian gravitational constant seems to reach a magnitude of $8.205 \times 10^{22}$ $\mathrm{m}^{3} \mathrm{~kg}^{-1} \mathrm{sec}^{-2}$.

With reference to the proposed assumptions and based on the above points, quantitatively, we noticed that, 


$$
G_{F} \cong\left[\left(G_{e} m_{p}^{2}\right)^{2}\left(G_{N} m_{p}^{2}\right)\right]^{\frac{1}{3}}\left(\frac{2 G_{s} m_{p}}{c^{2}}\right)^{2}
$$

Based on this relation,

$$
\begin{aligned}
G_{N} \cong \frac{G_{F}^{3} c^{12}}{64 G_{e}^{2} G_{s}^{6} m_{p}^{12}} \\
G_{N} \propto\left(\frac{G_{F}^{3}}{G_{e}^{2} G_{s}^{6}}\right)
\end{aligned}
$$

If, recommended $G_{F} \cong 1.435850984 \times 10^{-62} \mathrm{~J}^{3}{ }^{3}$, obtained $G_{N} \cong 6.619386 \times 10^{-11} \mathrm{~m}^{3} \mathrm{~kg}^{-1} \mathrm{sec}^{-2}$.

With reference to proposed assumptions, $G_{N}$ can be expressed with,

$$
\begin{aligned}
& G_{N} \cong\left(\frac{m_{e}}{m_{p}}\right)^{9}\left(\frac{G_{s}}{G_{e}}\right)\left(\frac{\hbar c}{m_{p}^{2}}\right) \\
& \cong 6.679856 \times 10^{-11} \mathrm{~m}^{3} \mathrm{~kg}^{-1} \mathrm{sec}^{-2}
\end{aligned}
$$

Based on relations (11) and (14),

If, $G_{s} \cong 3.32956081 \times 10^{28} \mathrm{~m}^{3} \mathrm{~kg}^{-1} \mathrm{sec}^{-2}$

$$
G_{F} \cong \frac{4 \hbar G_{s}^{2} m_{e}^{2}}{c^{3}} \cong 1.44021 \times 10^{-62} \mathrm{~J} . \mathrm{m}^{3}
$$

If, $G_{F} \cong 1.435850984 \times 10^{-62} \mathrm{~J} . \mathrm{m}^{3}$,

$$
G_{s} \cong \sqrt{\frac{G_{F} c^{3}}{4 \hbar m_{e}^{2}}} \cong 3.3245183 \times 10^{28} \mathrm{~m}^{3} \mathrm{~kg}^{-1} \mathrm{sec}^{-2}
$$

Electron rest mass can be fitted with,

$$
m_{e} \cong \sqrt{\frac{G_{F} c^{3}}{4 \hbar G_{s}^{2}}} \cong \frac{1}{2 G_{s}} \sqrt{\frac{G_{F} c^{3}}{\hbar}}
$$

\section{Nuclear Planck mass and its Schwarzschild radius}

With reference to Planck mass, nuclear Planck mass can be expressed with:

$$
m_{n p l} \cong \sqrt{\frac{\hbar c}{G_{s}}} \cong 546.62 \mathrm{MeV} / c^{2}
$$

With reference to Schwarzschild radius of a black hole, Schwarzschild radius of nuclear Planck mass can be expressed with:

$$
R_{n p l} \cong \frac{2 G_{s} m_{n p l}}{c^{2}} \cong \frac{2 G_{s}}{c^{2}} \sqrt{\frac{\hbar c}{G_{s}}} \cong 2 \sqrt{\frac{G_{s} \hbar}{c^{3}}} \cong 0.722 \mathrm{fm}
$$

Based on relations (15, 16 and 17),

$$
m_{e} \cong \sqrt{\frac{G_{F} c^{3}}{4 \hbar G_{s}^{2}}} \cong \frac{1}{R_{n p l}} \sqrt{\frac{G_{F}}{G_{s}}}
$$

\section{To understand proton's melting point}

With reference to Hawking black hole temperature formula [12], melting point of proton $[13,14]$ can be understood with:

$$
T_{\text {proton }} \cong \frac{\hbar c^{3}}{8 \pi k_{B} G_{s} m_{p}} \cong 0.15 \times 10^{12} \mathrm{~K}
$$

Based on this relation and with reference to up quark, other quark melting points can be expressed with the following kind of relation.

$$
T_{\text {quark }} \cong\left(\frac{m_{q}}{m_{u p}}\right) \frac{\hbar c^{3}}{8 \pi k_{B} G_{s} m_{u p}}
$$

where $\left(\frac{m_{q}}{m_{u p}}\right)$ represents the ratio of mass of any quark to mass of up quark. Based on this relation, for up quark of rest energy $2 \mathrm{MeV}$, its corresponding $T_{u p} \cong 69$ Tera $K$ and $8 \pi k_{B} T_{u p} \cong 236 \mathrm{MeV}$. This energy can be compared with the currently believed QCD energy scale, (170 to 270$) \mathrm{MeV}$ [14].

\section{To fit neutron's life time}

Neutron life time [15] can be fitted with:

$$
\begin{aligned}
& t_{n} \cong \sqrt{\frac{G_{e}}{G_{N}}}\left(\frac{G_{s} m_{n}^{2}}{\left(m_{n}-m_{p}\right) c^{3}}\right) \\
& \cong \sqrt{\frac{G_{s}}{G_{N}}}\left(\frac{\sqrt{G_{e} G_{s}} m_{n}^{2}}{\left(m_{n}-m_{p}\right) c^{3}}\right) \cong 896.8 \mathrm{sec}
\end{aligned}
$$

where, $m_{n}=$ Rest mass of neutron,

$\sqrt{\frac{G_{e}}{G_{N}}} \cong 5.9645176 \times 10^{23} \approx$ Avogadro number, $N_{A}[16]$

By considering the unified atomic mass unit,

$$
m_{\text {atom }} \cong 1.66054 \times 10^{-27} \mathrm{~kg} \text {, }
$$

$$
t_{n} \cong \sqrt{\frac{G_{s}}{G_{N}}}\left(\frac{\sqrt{G_{e} G_{s}} m_{\text {atom }}^{2}}{\left(m_{n}-m_{p}\right) c^{3}}\right) \cong 881.5 \mathrm{sec}
$$

This value can be compared with the recommended value [2] and results of bottle experiments [16]. 


\section{Understanding nuclear stability and binding energy}

\section{a) Proton-Neutron stability:}

Let,

$$
s \cong\left(\frac{G_{s} m_{p} m_{e}}{\hbar c}\right) \cong\left(\frac{\hbar c}{G_{e} m_{e}^{2}}\right) \cong 1.604637101 \times 10^{-3}
$$

Using this ratio, proton-neutron stability relation can be fitted directly in the following way [17].

$$
\begin{aligned}
& A_{s} \cong 2 Z+s(2 Z)^{2} \cong 2 Z+(4 s) Z^{2} \\
& \cong 2 Z+0.00641855 Z^{2}
\end{aligned}
$$

where $A_{s}$ is the estimated stable mass number of $Z$. It is very interesting to note that, close to beta stability line, considering $(4 s) \cong k \cong 0.00641855$, nuclear binding energy $[18,19]$ can be estimated very easily. One very interesting observation is that,

$$
\exp \left(\frac{m_{n}-m_{p}}{m_{e}}\right) \cong \sqrt{\frac{1}{4 s}} \cong \sqrt{\frac{1}{k}} \approx 4 \pi
$$

\section{b) Nuclear binding energy:}

$\alpha_{s}$ being the strong coupling constant [2], characteristic nuclear binding energy potential can be expressed with the following relation $[19,20]$.

$$
B_{0} \cong\left(\frac{1}{\alpha_{s}}\right)\left(\frac{e^{2}}{4 \pi \varepsilon_{0} R_{0}}\right) \cong\left(\frac{1}{\alpha_{s}}\right)\left(\frac{e^{2} c^{2}}{8 \pi \varepsilon_{0} G_{s} m_{p}}\right) \cong 10.09 \mathrm{MeV}
$$

Note: With reference to $\alpha_{s}$, it is possible to consider, $\left(\frac{G_{s} m_{p}^{2}}{\hbar c}\right)^{2} \cong \frac{1}{\alpha_{s}} \cong \frac{1}{0.1152}$. Considering $\left(\frac{G_{s} m_{p}^{2}}{\hbar c}\right) \cong 2.9464$, it is possible to define the existence of a strongly interacting nuclear elementary charge of magnitude, $e_{s} \cong\left(\frac{G_{s} m_{p}^{2}}{\hbar c}\right) e$. For details, readers are encouraged to see our preprint [21].

Based on the new integrated model proposed by $\mathrm{N}$. Ghahramany et al [18] and with reference to relation (26), for $Z \cong(40$ to 83$)$, close to the beta stability line, we noticed that,

$$
\left[\frac{N_{s}^{2}-Z^{2}}{Z}\right] \cong k Z A_{s}
$$

where $\left(A_{s}-Z\right)=N_{s}$.

$$
\begin{aligned}
(B)_{A_{s}} & \cong\left[A_{s}-\left(\frac{N_{s}^{2}-Z^{2}}{3 Z}\right)\right] \times 9.5 \mathrm{MeV} \\
& \cong\left[A_{s}-\left(\frac{k Z A_{s}}{3}\right)\right] \times 9.5 \mathrm{MeV}
\end{aligned}
$$

Based on this strange and simple relation and first four terms of the semi empirical mass formula (SEMF), close to the beta stability line, for $(Z=2$ to 100$)$, it is possible to show that,

$$
(B)_{A_{s}} \cong\left[A_{s}-A_{s}^{1 / 3}-\frac{k A_{s} \sqrt{N_{s} Z}}{3.40}-1\right] \times\left(B_{0} \cong 10.09 \mathrm{MeV}\right)
$$

For relation (31), see figure 1 (dashed red curve) for the estimated binding energy per nucleon close to the beta stability line of $Z=2$ to 100 compared with first four terms of the SEMF (Green curve) where: $a_{v} \cong 15.77 \mathrm{MeV}$, $a_{s} \cong 18.34 \mathrm{MeV}, a_{a} \cong 23.21 \mathrm{MeV}$ and $a_{c} \cong 0.71 \mathrm{MeV}$.

Figure 1: Binding energy per nucleon close to beta stability line from $Z=2$ to 100

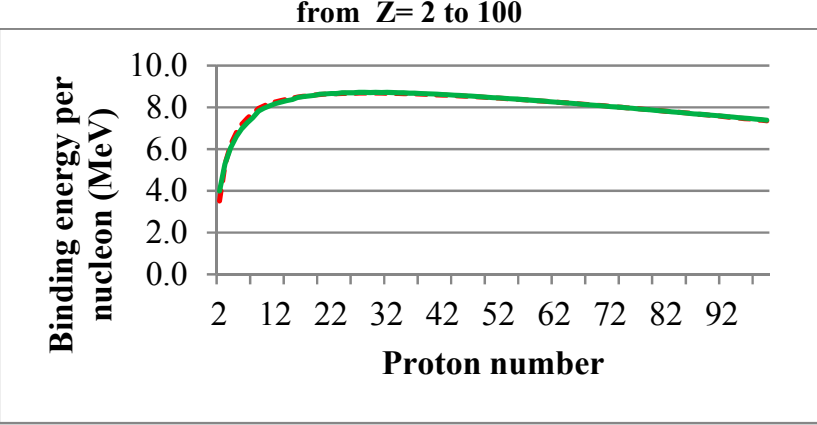

\section{Discussion and conclusion}

We appeal that,

a) We presented a number of applications connecting micro-macro physical systems and finally developed arithmetic relations for understanding the role of the Newtonian gravitational constant [22] in microscopic physics. Following this kind of computational approach, it is certainly possible to reproduce another set of arithmetic relations by using which, in near future; in a verifiable approach, it may be possible to find a set of absolute relations and $G_{N}$ can be estimated. For example, based on the recommended values of $R_{p} \cong 0.8751 \mathrm{fm}$ and $G_{F} \cong 1.435850984 \times 10^{-62}{\mathrm{~J} . \mathrm{m}^{3}}^{3}$,

$>$ With reference to relations (1,2 and 3 or 14), $G_{N} \cong 6.679856 \times 10^{-11} \mathrm{~m}^{3} \mathrm{~kg}^{-1} \mathrm{sec}^{-2}$.

$>$ With reference to relations (1, 5 and 14), $G_{N} \cong 6.6706246 \times 10^{-11} \mathrm{~m}^{3} \mathrm{~kg}^{-1} \mathrm{sec}^{-2}$. 
$>$ With reference to relations $(1,14$ and 16), $G_{N} \cong 6.6697396 \times 10^{-11} \mathrm{~m}^{3} \mathrm{~kg}^{-1} \mathrm{sec}^{-2}$.

$>$ With reference to relations $(1,3$, and 5), $G_{N} \cong 6.6660136 \times 10^{-11} \mathrm{~m}^{3} \mathrm{~kg}^{-1} \mathrm{sec}^{-2}$.

$>$ With reference to relations (1, 3 and 16), $G_{N} \cong 6.664687 \times 10^{-11} \mathrm{~m}^{3} \mathrm{~kg}^{-1} \mathrm{sec}^{-2}$.

$>$ With reference to relations (1, 2 and 12), $G_{N} \cong 6.619386 \times 10^{-11} \mathrm{~m}^{3} \mathrm{~kg}^{-1} \mathrm{sec}^{-2}$.

$>$ With reference to relations (1, 5 and 12), $G_{N} \cong 6.6745406 \times 10^{-11} \mathrm{~m}^{3} \mathrm{~kg}^{-1} \mathrm{sec}^{-2}$.

b) The ultimate aim of 'Ideas Lab' $\left(16^{\text {th }}\right.$ May to $26^{\text {th }}$ October 2016) [6] organized by the Physics Division of the Mathematical and Physical Sciences Directorate at the National Science Foundation (NSF), was to facilitate the development of new experiments designed to measure Newton's gravitational constant $G_{N}$ with relative uncertainties approaching or surpassing one part in 100,000 . In this context, we humbly and sincerely request NSF to consider and encourage our proposed method of estimating the Newtonian gravitational constant with possible support.

c) As it is inevitable to unite gravity and other three atomic interactions, if one is willing to explore the possibility of incorporating the proposed assumptions either in String theory models or in Quantum gravity models, certainly, background physics assumed to be connected with proposed semi empirical relations can be understood and in near future, a 'workable' or 'practical' model of "everything" can be developed. Based on relations (11) to (14), Fermi's weak coupling constant and the three gravitational constants can be fitted in a unified approach and finally, in a verifiable approach, Newtonian gravitational constant can be estimated accurately with microscopic physical constants.

\section{Acknowledgements}

Author Seshavatharam U.V.S is indebted to professors brahmasri Dr. M. Nagaphani Sarma, Chairman, shri K.V. Krishna Murthy, former chairman, Institute of Scientific Research in Vedas (ISERVE), Hyderabad, India and Shri K.V.R.S. Murthy, former scientist IICT (CSIR), Govt. of India, Director, Research and Development, I-SERVE, for their valuable guidance and great support in developing this subject.

\section{References}

[1] U. V. S. Seshavatharam and S. Lakshminarayana. Towards a workable model of final unification. International Journal of Mathematics and Physics Vol. 7, No. 1, 117 (2016). Al-Farabi Kazakh National University.

[2] C. Patrignani et al. (Particle Data Group), Chin. Phys. C, 40, 100001 (2016) and 2017 update
[3] Roberto Onofrio. On Weak Interactions as Short- Distance Manifestations of Gravity. Modern Physics Letters A, Vol. 28, No. 7, 1350022 (2013)

[4] G. Rosi, F. Sorrentino, L. Cacciapuoti, M. Prevedelli and G. M. Tino1. Precision measurement of the Newtonian gravitational constant using cold atoms. Nature 510, 518-521. (2014)

[5] S. Schlamminger and R.D. Newman. Recent measurements of the gravitational constant as a function of time. Phys. Rev. D 91, 121101 (2015)

[6] Ideas Lab: Measuring "Big G" Challenge. https://www.nsf.gov/pubs/2015/nsf15591/nsf15591.htm.

[7] K. Becker, M. Becker and J. H. Schwarz. String Theory and M-theory: A Modern Introduction. Cambridge University Press, (2006)

[8] Salam A, Sivaram C. Strong Gravity Approach to QCD and Confinement. Mod. Phys. Lett., v. A8(4), 321-326. (1993)

[9] O. F. Akinto, Farida Tahir. Strong Gravity Approach to QCD and General Relativity. arXiv:1606.06963v3

[10] N. Chamel et al. On the Maximum Mass of Neutron Stars. Int. J. Mod. Phys. E22 (2013) 1330018

[11] Sebastien Guillot et al. Measurement of the Radius of Neutron Stars with High S/N Quiescent Low-mass X-ray Binaries in Globular Clusters. Astrophys.J. 772 (2013).

[12] S. Hawking, Particle Creation by Black Hole, Commun. Math. Phys. 43, 199-220(1975)

[13] Helmut Satz. The Quark-Gluon Plasma.Nucl.Phys.A862863:4-12,2011.

[14] Horst Stoecker et al. Glueballs amass at RHIC and LHC Colliders! - The early quarkless 1 st order phase transition at $\mathrm{T}=270 \mathrm{MeV}$ - from pure Yang-Mills glue plasma to GlueBall-Hagedorn states. J. Phys. G 43 (2016) 1, 015105.

[15] C. L. Morris et al. A new method for measuring the neutron lifetime using an in situ neutron detector. Report number: LAUR-16-27352. https://arxiv:1610.04560.

[16] B. Andreas et al. An accurate determination of the Avogadro constant by counting the atoms in a $28 \mathrm{Si}$ crystal. Phys. Rev. Lett. 106, 030801.

[17] Chowdhury, P.R. et al. Modified Bethe-Weizsacker mass formula with isotonic shift and new driplines. Mod. Phys. Lett. A20 p.1605-1618. (2005).

[18] N.Ghahramany et al. New approach to nuclear binding energy in integrated nuclear model. Journal of Theoretical and Applied Physics 6:3 (2012).

[19] U. V. S Seshavatharam, S. Lakshminarayana. On the role of strong coupling constant and nucleons in understanding nuclear stability and binding energy. Journal of Nuclear Sciences. (2017); 4(1): 7-18.

[20] Seshavatharam, U.V.S.; Lakshminarayana, S.To unite nuclear and sub-nuclear strong interactions. International Journal of Physical Research, 5 (2) (2017) 104-108.

[21] Seshavatharam, U.V.S.; Lakshminarayana, S. To Develop a Virtual Model of Microscopic Quantum Gravity. Preprints 2017,2017110119, 23 pages. (doi: 10.20944/preprints201711.0119.v1).

[22] Brandenburg J. E. The GEM Unification Theory of the Vacuum: Did Dimensional Collapse Trigger the Big Bang. International Journal of Astrophysics and Space Science. Special Issue: Quantum Vacuum, Fundamental Arena of the Universe: Models, Applications and Perspectives. Vol. 2, No. 6-1, pp. 24-38. (2014). 